\title{
Control operacional para reducción de consumo de energía eléctrica en el Instituto Tecnológico de Chihuahua II
}

\section{Operational control to reduce electricity energy consumption at the Instituto Tecnológico de Chihuahua II}

HERNÁNDEZ-RODRÍGUEZ, María Guadalupe*†, ORTEGA-CHÁVEZ, Laura Antonia, RUIZRODRÍGUEZ, Irma Judith y CARO-ESCUDERO, Iveth Selene

Instituto Tecnológico de Chihuahua II / Tecnológico Nacional de México

ID $1^{\text {er }}$ Autor: Maria Guadalupe Hernández-Rodríguez / ORC ID: 0000-0001-7278-7699, Researcher ID Thomson: I-65412018

ID $1^{\text {er }}$ Coautor: Laura Antonia, Ortega-Chávez / ORC ID: 0000-0001-7860-1277

ID $2^{\text {do }}$ Coautor: Irma Judith, Ruiz-Rodríguez / ORC ID: 0000-0001-6566-3161

ID $3^{\text {er }}$ Coautor: Iveth Selene, Caro-Escudero / ORC ID: 0000-0002-3611-2309

DOI: $10.35429 /$ JEA.2019.21.6.24.33

Recibido: 19 de Junio 2019; Aceptado 30 de Septiembre, 2019

\section{Resumen}

El control operacional para reducción de consumo de energía eléctrica en el Instituto Tecnológico de Chihuahua II, es un tema de gran interés para la comunidad Tecnológica, ya que es una Institución comprometida con el cuidado del medio ambiente y el uso eficiente de la energía. Actualmente se encuentra certificado por la norma ISO 14001 en su Sistema de Gestión Ambiental (SGA) y la norma ISO 50001 en su Sistema de Gestión de la Energía (SGEn). Se cuenta con información de los consumos de energía eléctrica desde el año 2011, y es a partir del año 2012 que se implementó un programa de monitoreo y ayudas visuales para el control operacional del consumo y reducción de la misma. El objetivo de la investigación es determinar si las acciones tomadas están resultando en una reducción de los consumos y uso eficiente de energía eléctrica. De acuerdo a los datos considerados se observa un indicador a la baja, con una ligera alza en el 2018, determinándose si esta reducción es estadísticamente significativa, se realiza una serie de análisis estadísticos tales como ANOVA, regresión, correlación y pruebas pareadas.

\section{Control, Energía eléctrica, Reducción, Eficiencia}

\begin{abstract}
Operational control to reduce electricity consumption in the Technological Institute of Chihuahua II, is a subject of great interest for the technological community, as it is an institution committed to caring for the environment and the efficient use of energy. It is currently certified by the ISO 14001 standard in its Environmental Management System (SGA) and the ISO 50001 standard in its Energy Management System (SGEn). There is information on electricity consumption since 2011, and it is from 2012 that a monitoring program and visual aids for the operational control of consumption and its reduction were implemented. The objective of the investigation is to determine if the actions taken are resulting in a reduction of consumption and efficient use of electrical energy. According to the considered data, a downward indicator is observed, with a slight increase in 2018, determining if this reduction is statistically significant, a series of statistical analyzes is performed, such as ANOVA, regression, correlation and paired tests
\end{abstract}

Control, Electric power, Reduction, Efficiency

Citación: HERNÁNDEZ-RODRÍGUEZ, María Guadalupe, ORTEGA-CHÁVEZ, Laura Antonia, RUIZ-RODRÍGUEZ, Irma Judith y CARO-ESCUDERO, Iveth Selene. Control operacional para reducción de consumo de energía eléctrica en el Instituto Tecnológico de Chihuahua II. Revista de Aplicaciones de la Ingeniería. 2019. 6-21: 24-33

\footnotetext{
* Correspondencia del Autor (Correo electrónico: maria.hr@chihuahua2.tecnm.mx)

$\dagger$ Investigador contribuyendo como primer autor.
} 


\section{Introducción}

Actualmente el uso de la electricidad es fundamental para realizar gran parte de nuestras actividades; gracias a este tipo de energía tenemos una mejor calidad de vida. Con tan solo oprimir botones obtenemos luz, calor, frío, imagen o sonido. Su uso es indispensable y difícilmente nos detenemos a pensar acerca de su importancia y de los beneficios al utilizarla eficientemente.

El ahorro de energía eléctrica es un elemento fundamental para el aprovechamiento de los recursos energéticos; ahorrar equivale a disminuir el consumo de combustibles en la generación de electricidad evitando también la emisión de gases contaminantes hacia la atmósfera.

Nuestro país posee una gran cantidad de fuentes de energía. La mayor parte de la generación de electricidad se realiza a través del petróleo, carbón y gas natural, impactando de manera importante el medio ambiente al depender de los recursos no renovables, como son los combustibles fósiles. Al utilizarlos se emite a la atmósfera una gran cantidad de gases de efecto invernadero, los cuales, provocan el calentamiento global de la tierra, cuyos efectos se están manifestando y son devastadores.

Ahorrar y usar eficientemente la energía eléctrica, así como cuidar el medio ambiente, no son sinónimo de sacrificar o reducir nuestro nivel de bienestar o el grado de satisfacción de nuestras necesidades cotidianas, por el contrario, un cambio de hábitos y actitudes pueden favorecer una mayor eficiencia en el uso de la electricidad, el empleo racional de los recursos energéticos, la protección de la economía familiar y la preservación de nuestro entorno natural.

El Instituto Tecnológico de Chihuahua II no está ajeno a esta problemática que nos encontramos viviendo, es por ello que se encuentra certificado en las normas ISO 14001 para el Sistema de Gestión Ambiental e ISO 50001 para el Sistema de Gestión de la Energía, y constantemente se encuentra en la búsqueda de estrategias como el manejo de un control operacional con el cual se pretende disminuir el consumo de energía eléctrica y concientizar al personal docente, administrativo, técnico, intendentes y alumnos en el uso eficiente de la energía eléctrica.
Así como determinar si el uso de monitoreos y ayudas visuales en el Instituto Tecnológico de Chihuahua II como parte de este control, impactan en una reducción del consumo de energía eléctrica, siendo todo esto el objetivo principal de la investigación.

\section{Marco Teórico}

En los últimos años el consumo de energía eléctrica se ha incrementado a un ritmo acelerado, y debido a este ritmo de crecimiento se deben tomar una serie de acciones que impidan que aumente el índice físico del consumo energético. Sin embargo, no es fácil percibir que se está malgastando la energía eléctrica cuando se deja encendida una lámpara, la computadora, la televisión, un video juego, el aire acondicionado o la calefacción cuando no hay nadie en la habitación o se dejan conectados aparatos que no están en uso.

Esta realidad pone de manifiesto que la electricidad no es sólo ese enchufe donde se conectan los equipos, es el final de la inmensa cadena que se origina en las grandes centrales de generación y para que llegue hasta un hogar debe: ser generada en grandes y costosas plantas, en el mismo instante en que se requiera; transportada hasta los centros poblados, recorriendo muchos kilómetros y utilizando inmensas torres, transformadores, y cantidades de cables; distribuida en menores bloques de energía, hasta su hogar, utilizando cientos de transformadores, postes y kilómetros de cable; entregada, medida y facturada, para lo cual se requiere de equipos de medición, herramientas, personal para emitir y entregar facturas, así como para atender reclamos y solicitudes.

Todo este sistema eléctrico debe mantenerse al día, lo cual requiere personal especializado y alta tecnología en materiales y equipos. En vista de esto se están emprendiendo planes, programas económicos y energéticos, con la finalidad de aumentar las reservas existentes y combatir el uso desproporcionado que se tiene de la energía eléctrica.

Para definir las bases de los criterios necesarios en un programa de planeación energética, se requiere contar con escenarios del balance energético del país, conocer la oferta de energía "probada" y decidir el desarrollo económico y social del país que queremos tener.

HERNÁNDEZ-RODRÍGUEZ, María Guadalupe, ORTEGA-CHÁVEZ, Laura Antonia, RUIZ-RODRÍGUEZ, Irma Judith y CARO-ESCUDERO, Iveth Selene. Control operacional para reducción de consumo de energía eléctrica en el Instituto Tecnológico de Chihuahua II. Revista de Aplicaciones de la Ingeniería. 2019. 
La elaboración de balances energéticos requiere una gran cantidad de datos, lo que dificulta su realización. Aún más complejo es el establecimiento de escenarios futuros, ya que es necesario conocer cómo se desarrollarán diversos parámetros económicos, tecnológicos, políticos y comerciales, entre otros, así como suponer la evolución de la interrelación existente entre dichos parámetros.

Existen diferentes métodos para hacer planeación energética. Por ejemplo, el llamado LEAP, de las siglas en inglés para "planeación a largo alcance de energías alternativas" (Manzini et al, 1999), es un modelo que permite evaluar el efecto de diferentes políticas energéticas en el consumo de energía y en sus consecuentes emisiones.

Dentro de este esquema se calcula la energía consumida por la demanda, a partir de la energía gastada por los diferentes sectores finales. Se debe siempre considerar, además, que un programa de ahorro de energía repercutiría muy favorablemente en los requerimientos nacionales de inversión, divisas y desarrollo tecnológico

\section{Materiales y Métodos}

La población bajo estudio fue el instituto tecnológico de chihuahua II, debido a que se observó la necesidad de conocer si el monitoreo de aulas y las ayudas visuales que se llevan realizando desde el año 2011 como parte del control operacional contribuye a la reducción del consumo de energía eléctrica y un uso más eficiente de la misma.

Los datos para el análisis fueron proporcionados por el departamento del Sistema de Gestión Ambiental del mismo Instituto. Los métodos estadísticos utilizados para realizar el análisis fueron la prueba t-Student, pruebas pareadas y ANOVA, así como pruebas de hipótesis para diferencia de medias. Todos estos métodos fueron analizados y comprobados en el software de MINITAB 18 y EXCEL.

\section{Procedimiento}

Se recolectaron datos mensuales de los tres medidores con los que cuenta el Instituto Tecnológico de Chihuahua II para cada una de sus áreas, las cuales se muestran en la tabla 1.

\begin{tabular}{|l|l|}
\multicolumn{2}{c|}{$\begin{array}{c}\text { Clave del } \\
\text { medidor }\end{array}$} \\
\hline Y574M8 & $\begin{array}{l}\text { Edificios que alimenta } \\
\text { Vinculación, Idiomas, Desarrollo Académico, } \\
\text { Sistemas, Industrial, Biblioteca, C. de Computo } \\
\text { Taller de Industrial y edificios. B, D, E, F, G }\end{array}$ \\
\hline 579AJ8 & M, O, R \\
\hline 9E2H94 & Gimnasio \\
\hline
\end{tabular}

Tabla 1 Medidor y Área que alimenta

Fuente: Elaboración Propia

Se seleccionaron las mediciones de consumo de energía eléctrica del año 2010 y del año 2018, ya que en el año 2010 aún no se realizaba el monitoreo y fue hasta el siguiente año en el 2011 cuando se implementó el monitoreo y las ayudas visuales con el fin de controlar y disminuir el consumo de energía eléctrica. Dicha selección se realizó para comparar un año en el que no se efectuaba el monitoreo contra el año más actual en el que ya se efectúa el monitoreo, con el fin de determinar si hay diferencia significativa entre ellos con respecto al consumo de energía eléctrica.

El monitoreo lo realizan un grupo de alumnos del Instituto Tecnológico de Chihuahua II, como parte de las actividades del departamento de Desarrollo Académico en su área del Sistema de Gestión ambiental para otorgar créditos complementarios. Cada semestre se integra un grupo de aproximadamente 100 monitores, dos monitores por hora, 20 monitores por mes. El monitoreo consiste en pasar por los edificios y apagar las luces, minisplits y aire acondicionado cuando no se está impartiendo clase, o si los salones se encuentran vacíos, e ir registrando dichas incidencias en una hoja de reporte.

Los monitores tienen que presentarse con el coordinador del control de energía eléctrica del SGA semanalmente, para que este registre su firma en el reporte de control de uso eficiente de focos, minisplits y aire acondicionado, esto con el fin de llevar un control de las actividades de los alumnos. Con los consumos de energía eléctrica, se realizó un análisis de ANOVA mediante el paquete de Excel y Minitab 18, además se realizó un análisis de prueba de hipótesis para diferencia de medias y pruebas pareadas. Con este análisis se pretende determinar si existe efecto de los monitores y las ayudas visuales en la disminución del consumo de energía eléctrica en el Instituto Tecnológico de Chihuahua II. 


\section{Análisis de los datos}

Como ya se había mencionado anteriormente, el análisis se realizó con datos de año 2010 y 2018. Con ello se pretende averiguar si existen diferencias significativas entre ambos grupos a nivel de consumo de energía eléctrica. Las pruebas estadísticas que se eligieron son aquellas que permiten comparar las medias de una variable continua entre dos o más grupos, estas pruebas son la t-student, las pruebas pareadas y la ANOVA.

Cada una de estas pruebas ha sido diseñada para poder ser aplicada cuando se cumplen una serie de supuestos necesarios, bajo diferentes condiciones de aplicación, como: La normalidad en la distribución de la variable, la homocedasticidad (igualdad de varianzas), y la independencia de las observaciones. Se analizó primeramente el área de Recursos Materiales la cual presentó los consumos que se muestran en la tabla 2.

\begin{tabular}{|l|r|r|r|}
\hline \multicolumn{4}{|c|}{ Recursos Materiales } \\
\hline & Consumo & & \\
\hline Mes & 2010 & 2018 & Diferencia \\
\hline Enero & 64680 & 60,624 & $-4,056$ \\
\hline Febrero & 76008 & 51,192 & $-24,816$ \\
\hline Marzo & 78432 & 43,704 & $-34,728$ \\
\hline Abril & 67080 & 54,120 & $-12,960$ \\
\hline Mayo & 67608 & 90,408 & 22,800 \\
\hline Junio & 93984 & 82,646 & $-11,338$ \\
\hline Julio & 76224 & 41,352 & $-34,872$ \\
\hline Agosto & 53208 & 82,464 & 29,256 \\
\hline Septiembre & 136896 & 70,824 & $-66,072$ \\
\hline Octubre & 91224 & 54,288 & $-36,936$ \\
\hline Noviembre & 83352 & 61,368 & $-21,984$ \\
\hline Diciembre & 70392 & 55,296 & $-15,096$ \\
\hline
\end{tabular}

Tabla 2 Consumo mensual de energía eléctrica en los años 2010 y 2018

Fuente: Elaboración Propia

El test de la razón de varianzas o test de Levene, es un método estadístico que nos permitirá decidir si la varianza en ambos grupos es o no la misma. Si su p-valor es menor a 0.05 , rechazaremos la hipótesis nula y supondremos que la variabilidad en ambos grupos es sustancialmente distinta (varianzas no homogéneas), y si su p-valor es mayor o igual a 0.05 , no rechazaremos la hipótesis nula y asumiremos igualdad de varianzas. Para este estudio dichos cálculos se realizaron en Minitab, al igual que la prueba de normalidad.
Como se puede observar en la gráfica 1 , basados en una distribución normal, la prueba $\mathrm{F}$ para nuestro estudio, presenta una $\mathrm{p}$-valor de 0.348 la cual es mayor a 0.05 , por lo tanto, no se rechaza la hipótesis nula la cual supone igualdad de varianzas.

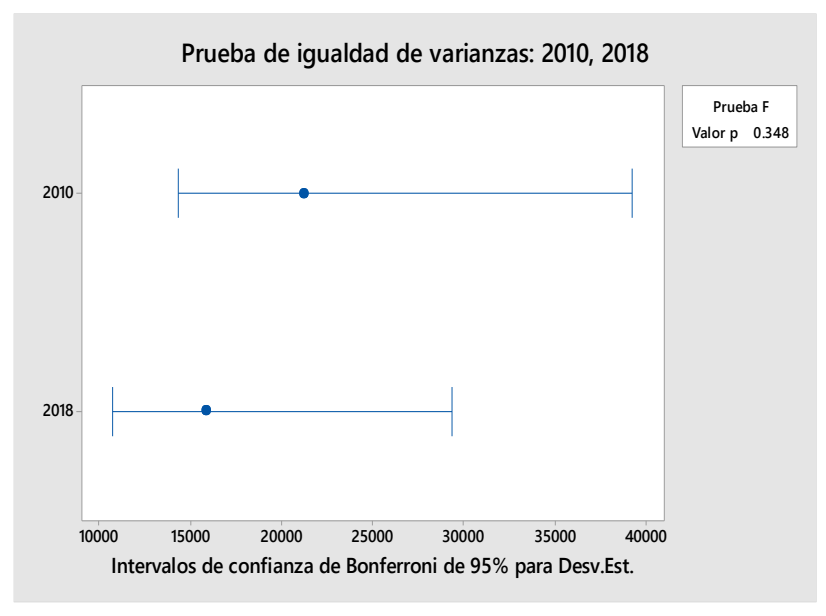

Gráfica 1 Prueba de igualdad de varianzas suponiendo una distribución normal de los años 2010 y 2018

Fuente: Elaboración Propia

La gráfica 2, presenta los resultados la prueba de igualdad de varianzas por medio de la prueba de Levene la cual nos da un p-valor de 0.851 , y por comparaciones múltiples la cual nos arroja un p-valor de 0.558 , en ambos casos el pvalor es mayor que 0.05 , por lo tanto, no se rechaza la hipótesis nula, la cual supone igualdad de varianzas entre las dos muestras.

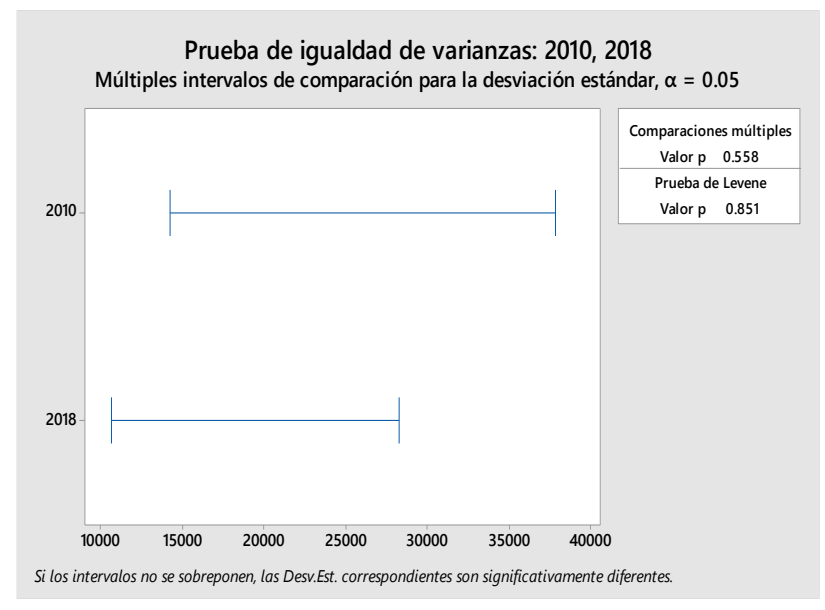

Gráfica 2 Prueba Levene para igualdad de varianzas entre el año 2010 y 2018

Fuente: Elaboración Propia

En la gráfica 3 y 4 se puede observar que los datos están relativamente cerca de la línea de distribución normal ajustada. 
El valor de p para los datos del año 2010 es menor que el nivel de significancia de 0.05 , por lo tanto, los dados en este caso no siguen una distribución normal, mientras que para el año 2018 el valor de $\mathrm{p}$ es mayor que 0.05 , lo cual implica que los datos siguen una distribución normal para el consumo de energía eléctrica.

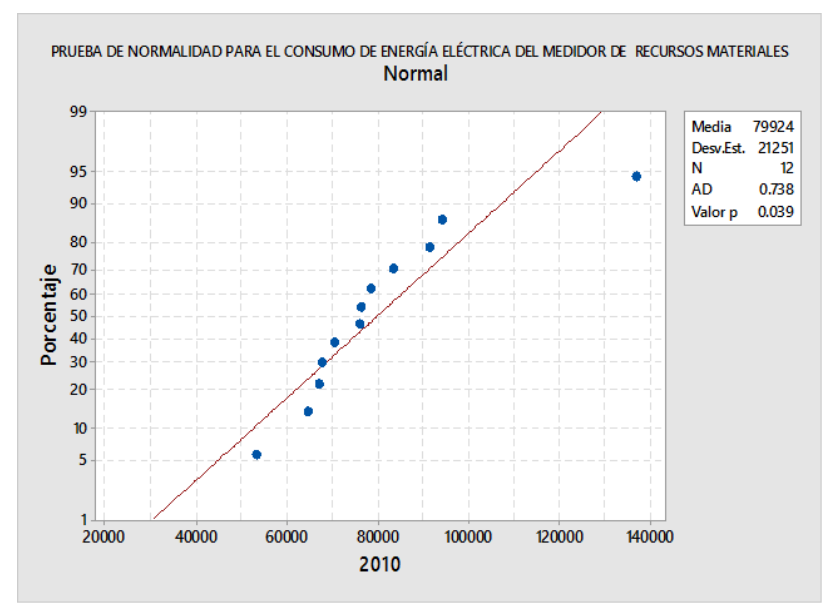

Gráfica 3 Prueba de normalidad para él consumo de energía eléctrica del medidor de recursos materiales del año 2010

Fuente: Elaboración Propia

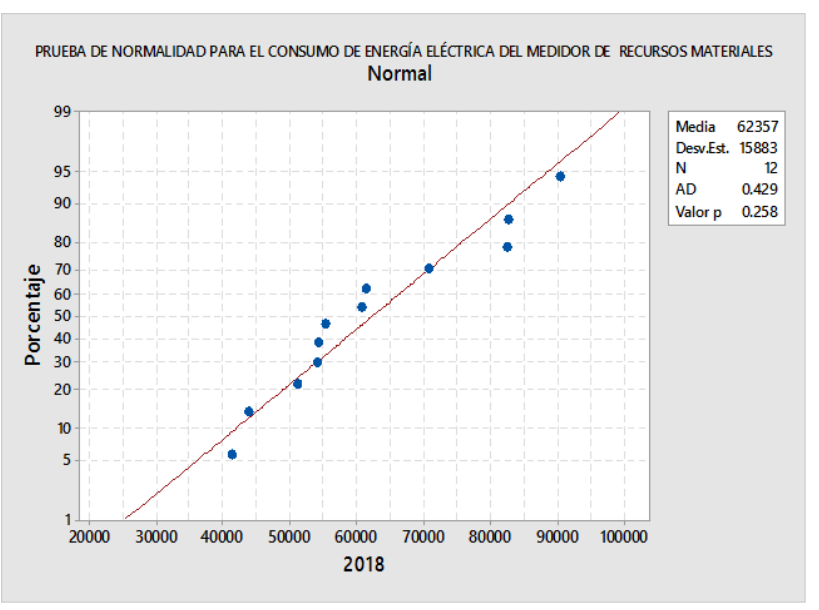

Gráfica 4 Prueba de normalidad para él consumo de energía eléctrica del medidor de recursos materiales del año 2018. Fuente: Elaboración Propia

\section{Análisis de ANOVA}

Se realizó el análisis ANOVA en forma manual como se muestra en la tabla 3, y en Minitab como se muestra en el cuadro 1 .

SCTratamientos $=\sum \frac{Y i^{2}}{n i}-\frac{Y^{2} . .}{\sum_{i=1}^{t} n i}=1,851,561,800$

$S C T=\sum_{J=1}^{t} \sum_{j=1}^{n i} Y^{2} i j-\frac{Y^{2} . .}{\sum_{i=1}^{t} n i}=9,594,463,276$

$S C E=S C T-$ SCTratamientos $=7,742,901,746$

\begin{tabular}{|l|l|l|l|l|l|}
\hline FV & GL & SC & CM & & $\mathrm{F}_{\mathrm{n}}^{\mathrm{m}} \alpha$ \\
& & & & $\mathrm{F}$ & \\
\hline TRATAMIENTOS & 1 & 1851561800 & 1851561800 & 5.26 & 4.30 \\
\hline ERROR & 22 & 7742901476 & 351950067.1 & & \\
\hline TOTAL & 23 & 9594463276 & & & \\
\hline
\end{tabular}

Tabla 3 Análisis ANOVA para el medidor Y574M8.

Fuente: Elaboración Propia

\begin{tabular}{|c|c|c|c|c|c|}
\hline \multicolumn{6}{|c|}{ One-way ANOVA: 2010, 2018} \\
\hline \multicolumn{3}{|c|}{$\begin{array}{l}\text { Null hypothesis } \\
\text { Alternative hypothesis } \\
\text { Significance level }\end{array}$} & \multicolumn{3}{|c|}{$\begin{array}{l}\text { All means are equal } \\
\text { Not all means are equal } \\
\alpha=0.05\end{array}$} \\
\hline \multicolumn{6}{|c|}{ Equal variances were assumed for the analysis. } \\
\hline \multicolumn{6}{|c|}{ Factor Information } \\
\hline Factor & Les & Valu & \multirow{2}{*}{\multicolumn{3}{|c|}{$\frac{\text { Values }}{2010,2018}$}} \\
\hline Factor & 2 & 2010 & & & \\
\hline \multicolumn{6}{|c|}{ Analysis of Variance } \\
\hline Source & DF & AdjjSS & AdjMS & F-Value $\mathrm{F}$ & P-Value \\
\hline Factor & 1 & 1851561800 & 1851561800 & 5.26 & 0.032 \\
\hline Error & 22 & 7742901476 & 351950067 & & \\
\hline Total & 23 & 9594463276 & & & \\
\hline
\end{tabular}

Cuadro 1 Análisis ANOVA para el medidor Y574M8 en MINITAB

Fuente: Elaboración Propia

Ha; Existe efecto de los monitores y ayudas visuales en la disminución del consumo de la energía eléctrica.

Ho; No existe efecto de los monitores y ayudas visuales en la disminución del consumo de energía eléctrica.

\section{$\mathrm{Ha} ; \quad \mu_{1} \neq \mu_{2}$ \\ Ho; $\mu_{1}=\mu_{2}$}

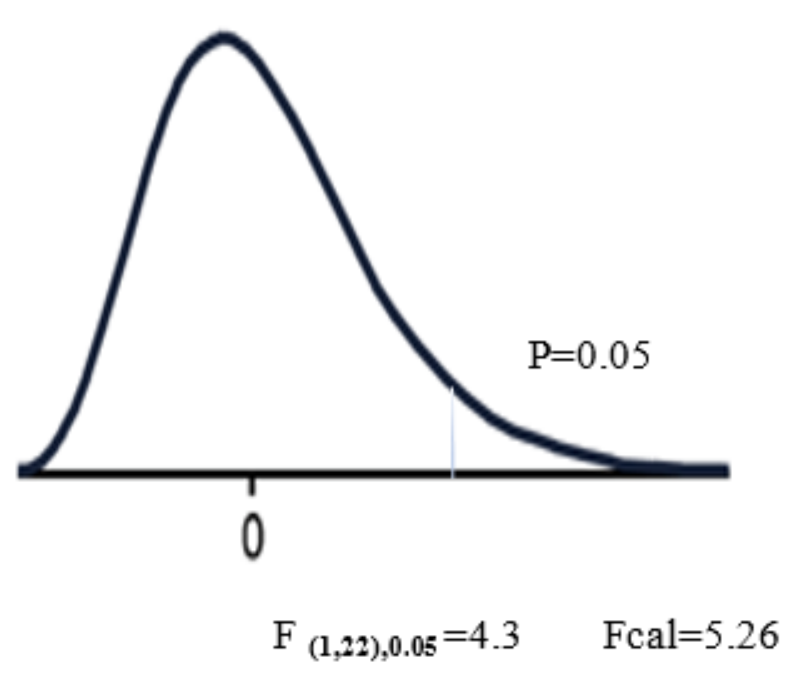

Figura 1 Distribución $\mathrm{F}$ para el medidor Y574M8. Fuente: Elaboración Propia 
En la figura 1 se puede observar que como la "F" calculada es mayor que la "F" de tabla, se rechaza Ho, es decir, existe evidencia suficiente para decir que el uso de monitores y ayudas visuales tienen efecto en la disminución del consumo de energía eléctrica.

\section{Análisis de la distribución muestral de diferencia de medias}

\begin{tabular}{|l|r|r|}
\hline & Año 2010 & Año 2018 \\
\hline $\mathrm{n}$ & 12 & 12 \\
\hline $\bar{x}$ & 79924 & 62357 \\
\hline $\mathrm{s}$ & 21251.353 & 15883.329 \\
\hline
\end{tabular}

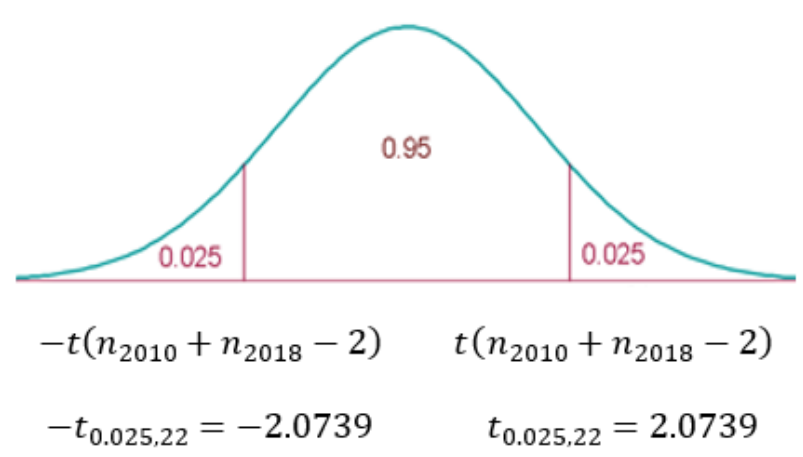

Figura 2 Distribución T-Student para diferencia de medias del medidor Y574M8. Fuente: Elaboración Propia

$\begin{array}{ll}H o ; & \mu_{2010}=\mu_{2018} \\ H a ; & \mu_{2010} \neq \mu_{2018}\end{array}$

\section{Regla de decision}

$H o ;-2.0739 \leq t c \leq 2.0739$

$H a ;-2.0739>t c \quad o \quad t c>2.0739$

$t_{c}=\frac{\left(\bar{x}_{2010}-\bar{x}_{2018}\right)-\left(\mu_{2010}-\mu_{2018}\right)}{\sqrt{S C^{2}\left(\frac{1}{n_{2010}}+\frac{1}{n_{2018}}\right)}}$

$t_{c}=\frac{(79924-62357.1667)}{\sqrt{(351950067)\left(\frac{1}{12}+\frac{1}{12}\right)}}=2.2936576$

$S C^{2}=\frac{\left(n_{2010}-1\right)\left(S_{2010}\right)^{2}+\left(n_{2018}-1\right)\left(S_{2018}\right)^{2}}{n_{2010}+n_{2018}-2}$

$S C^{2}=351950067$

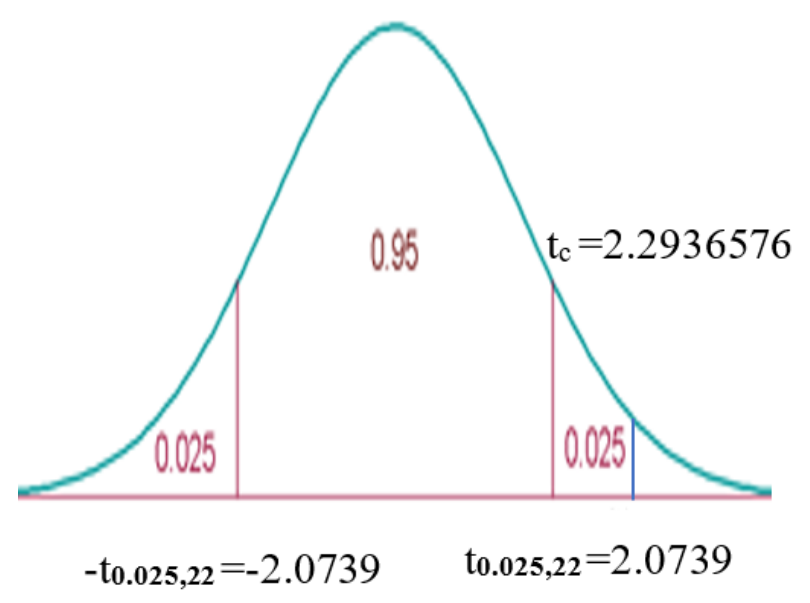

Figura 3 Comparación de valores de t de tabla, con la t calculada

Fuente: Elaboración Propia

$H_{o} ; \quad-2.0739 \leq 2.2936576 \leq 2.0739$

$H_{a} ;-2.0739>2.2936576$

$2.2936576>2.073$

Los datos muestran evidencia suficiente de que existe efecto de los monitores y ayudas visuales en la disminución del consumo de energía eléctrica. Los siguientes datos muestran el análisis en Minitab de la prueba T.

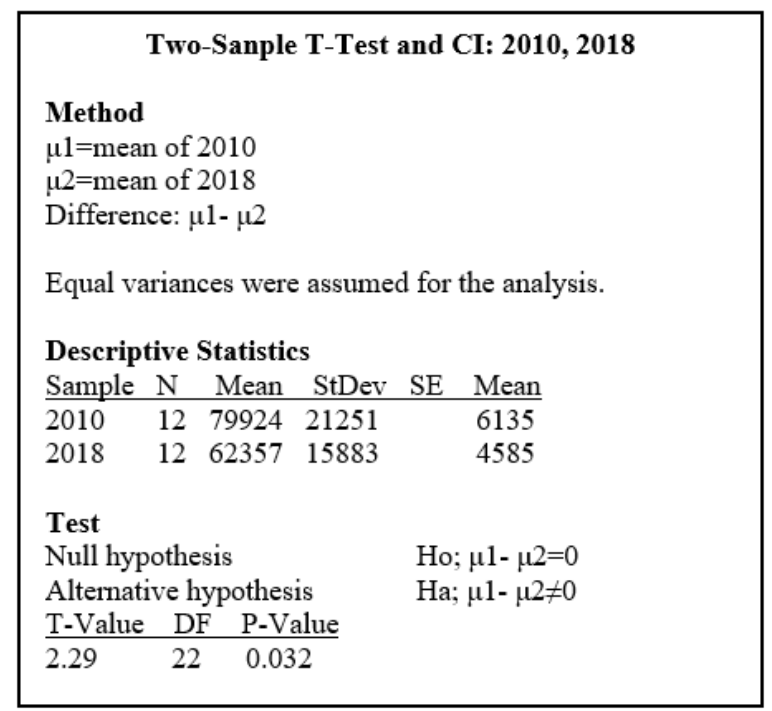

Cuadro 2 Análisis T-Student para el medidor Y574M8 en MINITAB

Fuente: Elaboración Propia

\section{Análisis de Pruebas Pareadas}

$$
\begin{aligned}
& H o ; \mu_{D}=0 \\
& \alpha=0.05 \\
& \sum_{\alpha} D=-210,802 \\
& \frac{\alpha}{2}=0.025 \\
& \bar{D}=-17,567 \\
& K=\text { Diferencial esperada }=0 \\
& \sum D^{2}=11,167,603,492
\end{aligned}
$$

HERNÁNDEZ-RODRÍGUEZ, María Guadalupe, ORTEGA-CHÁVEZ, Laura Antonia, RUIZ-RODRÍGUEZ, Irma Judith y CARO-ESCUDERO, Iveth Selene. Control operacional para reducción de consumo de energía eléctrica en el Instituto Tecnológico de Chihuahua II. Revista de Aplicaciones de la Ingeniería. 2019. 


$$
\begin{aligned}
& H o ; \mu_{D} \\
& a ; \mu_{D} \\
& t_{\alpha / 2,(n-1)}=t_{0.025,11}=2.201 \mathrm{M} \\
& S_{D}^{2}=678,589,081.1 \\
& S_{D}=26,049.74244 \\
& t_{c}=\frac{\sqrt{n} *(\bar{D}-k)}{S_{D}} \\
& t_{c}=\frac{\sqrt{12} *(-17,567-0)}{26,049.742}=-2.34 \\
& S_{D}^{2}=\frac{1}{n-1}\left(\sum D^{2}-\frac{\left(\sum D\right)^{2}}{n}\right)
\end{aligned}
$$

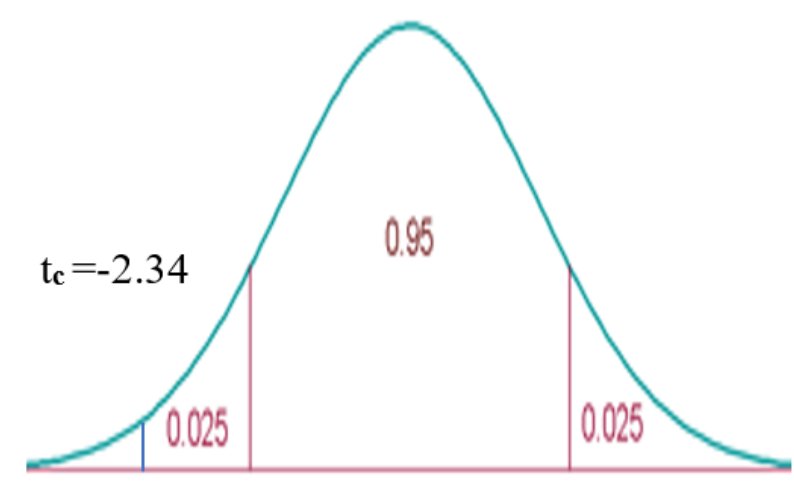

$$
-\mathrm{t}=-2.201 \quad \mathrm{~K}=0 \quad \mathrm{t}=2.201
$$

Figura 4 Comparación de valores de $\mathrm{t}$ de tabla, con la $\mathrm{t}$ calculada para pruebas pareadas

Fuente: Elaboración Propia

Se rechaza Ho, ya que $t_{c}<t_{\alpha / 2,(n-1)}$, es decir, existe evidencia suficiente para determinar que hay efecto de los monitores y ayudas visuales para la disminución del consumo de energía eléctrica.

\section{Análisis de Pruebas Pareadas en Minitab}

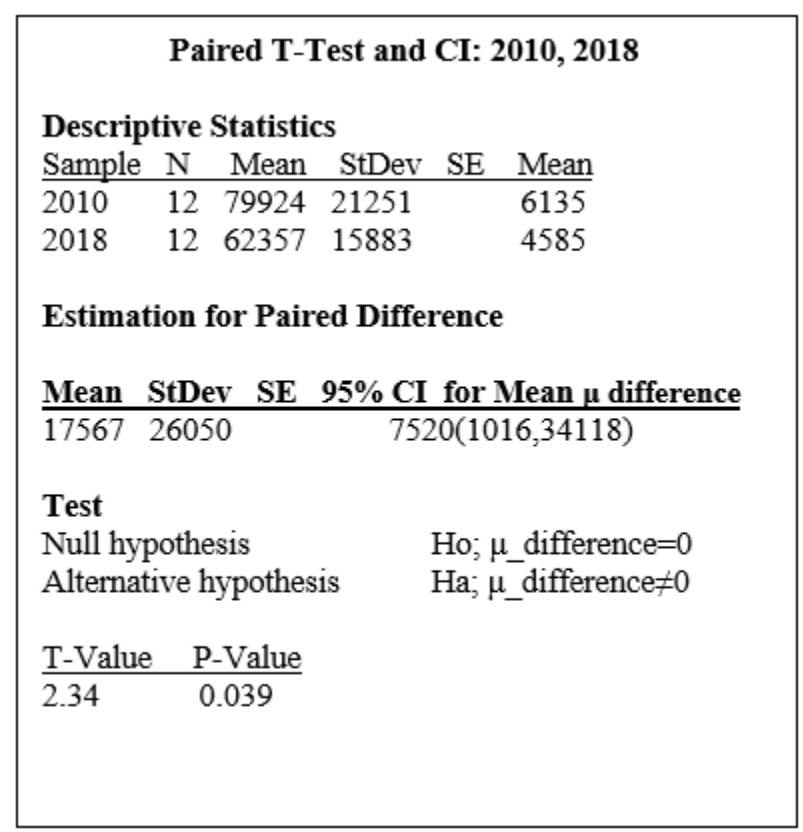

Cuadro 3 Análisis de Pruebas Pareadas para el medidor Y574M8 en MINITAB

Fuente: Elaboración Propia

ISSN 2410-3454

ECORFAN $®$ Todos los derechos reservados

\section{Análisis del área de los edificios MOR}

La siguiente tabla muestra los datos del consumo de energía eléctrica del medidor 579AJ8, durante los años 2010 y 1018.

\begin{tabular}{|l|r|r|r|}
\hline \multicolumn{4}{|c|}{ MOR } \\
\hline \multicolumn{1}{|c|}{ Mes } & \multicolumn{2}{|c|}{$\mathbf{2 0 1 0}$} & \multicolumn{2}{c|}{$\mathbf{2 0 1 8}$} & \multicolumn{1}{c|}{ Diferencia } \\
\hline Enero & 16944 & 16731 & -213 \\
\hline Febrero & 15504 & 13218 & $-2,286$ \\
\hline Marzo & 21408 & 12148 & $-9,260$ \\
\hline Abril & 17712 & 15967 & $-1,745$ \\
\hline Mayo & 12672 & 33053 & 20,381 \\
\hline Junio & 32760 & 29836 & $-2,924$ \\
\hline Julio & 42888 & 19840 & $-23,048$ \\
\hline Agosto & 38736 & 28597 & $-10,139$ \\
\hline Septiembre & 42048 & 27608 & $-14,440$ \\
\hline Octubre & 41304 & 19569 & $-21,735$ \\
\hline Noviembre & 28416 & 16354 & $-12,062$ \\
\hline Diciembre & 17472 & 13135 & $-4,337$ \\
\hline
\end{tabular}

Tabla 4 Consumo mensual de energía eléctrica en los años 2010 y 2018

Fuente: Elaboración Propia

En la siguiente gráfica se presenta la prueba de normalidad en Minitab para ambas muestras, y se puede observar que los datos siguen una distribución normal, ya que sus pvalor son mayores a 0.05 .

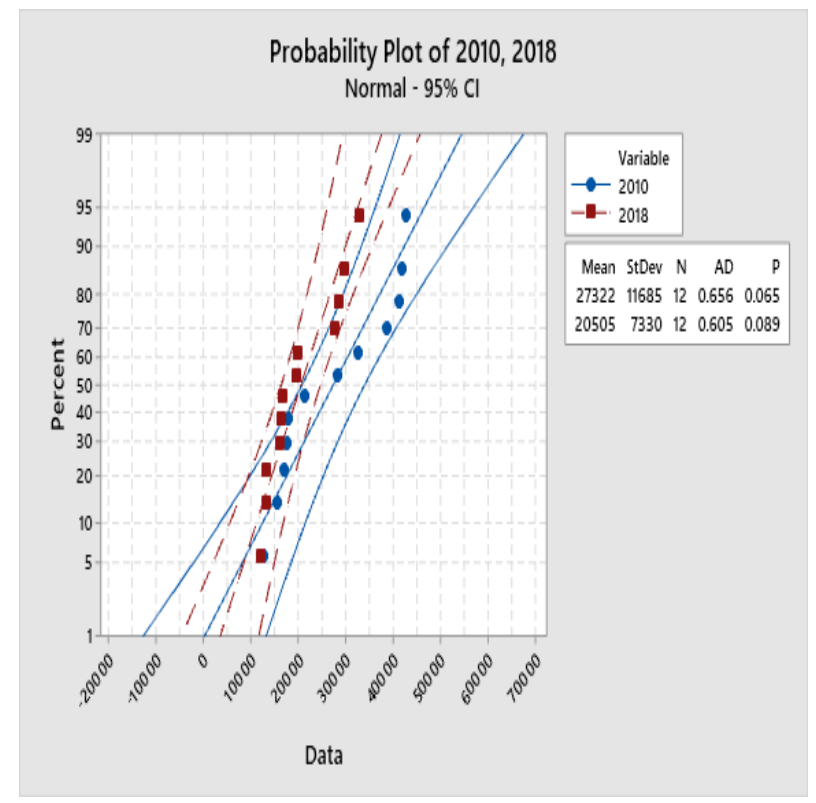

Gráfica 5 Prueba de normalidad para él consumo de energía eléctrica del medidor de los edificios MOR Fuente: Elaboración Propia

La prueba de igualdad de varianzas se realizó en Minitab por medio de la prueba $\mathrm{F}$, arrojando los siguientes resultados.

HERNÁNDEZ-RODRÍGUEZ, María Guadalupe, ORTEGA-CHÁVEZ, Laura Antonia, RUIZ-RODRÍGUEZ, Irma Judith y CARO-ESCUDERO, Iveth Selene. Control operacional para reducción de consumo de energía eléctrica en el Instituto Tecnológico de Chihuahua II. Revista de Aplicaciones de la Ingeniería. 2019. 


\begin{tabular}{|c|c|}
\hline \multicolumn{2}{|c|}{ Test Equal Variance } \\
\hline \multicolumn{2}{|l|}{ Method } \\
\hline Null hypothesis & All means are equal \\
\hline Alternative hypothesis & At least one variance is different \\
\hline Significance level & $\alpha=0.05$ \\
\hline \multicolumn{2}{|c|}{$\begin{array}{l}\text { F method is used. This method is accurate for normal data } \\
\text { only. }\end{array}$} \\
\hline Method Test Statistic & P-Value \\
\hline 2.54 & 0.137 \\
\hline
\end{tabular}

Cuadro 4 Análisis de Igualdad de varianzas para el medidor 579AJ8 en MINITAB

Fuente: Elaboración Propia

Como se puede observar, basados en una distribución normal, la prueba $\mathrm{F}$ para nuestro estudio, presenta una p-valor de 0.137 la cual es mayor a 0.05 , por lo tanto, no se rechaza la hipótesis nula la cual supone igualdad de varianzas.

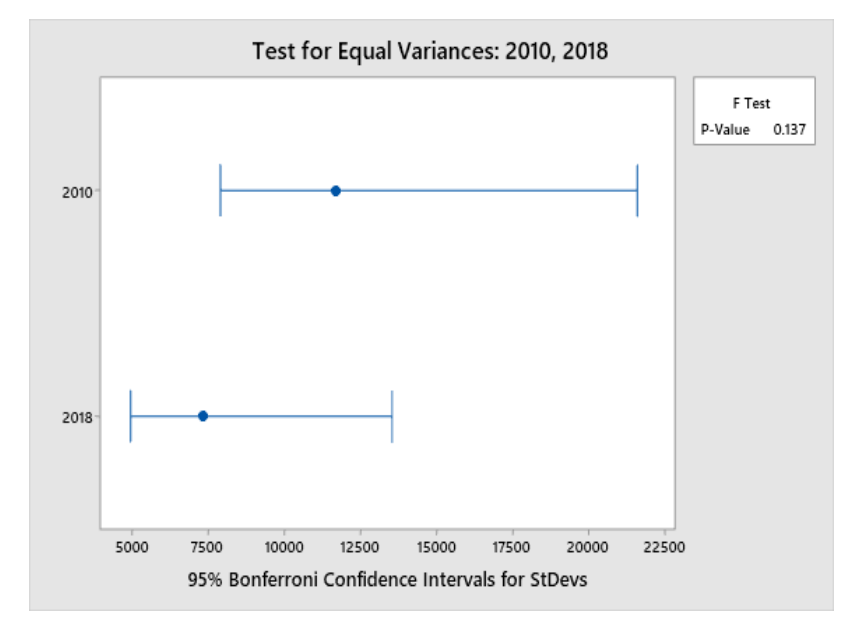

Gráfica 6 Prueba de igualdad de varianzas suponiendo una distribución normal de los años 2010 y 2018

Fuente: Elaboración Propia

\section{Análisis de ANOVA}

Ha; Existe efecto de los monitores y ayudas visuales en la disminución del consumo de la energía eléctrica.

Ho; No existe efecto de los monitores y ayudas visuales en la disminución del consumo de energía eléctrica.

Ha; $\mu_{1} \neq \mu_{2}$

Ho; $\mu_{1}=\mu_{2}$

SCTratamientos $=\sum \frac{Y i^{2}}{n i}-\frac{Y^{2} .}{\sum_{i=1}^{t} n i}=278,856,202.7$

$S C T=\sum_{J=1}^{t} \sum_{j=1}^{n i} Y^{2} i j-\frac{Y^{2} . .}{\sum_{i=1}^{t} n i}=2,371,991,835$

$S C E=S C T-$ SCTratamientos $=2093135633$

\begin{tabular}{|l|l|l|l|l|l|}
\hline \multicolumn{5}{|c|}{ ANOVA } \\
\hline FV & GL & SC & CM & F & $\mathrm{F}_{\mathrm{n}}^{\mathrm{m}} \alpha$ \\
\hline TRATAMIENTOS & 1 & 278856203 & 278856203 & 2.93 & 4.30 \\
\hline ERROR & 22 & 2093135633 & 95142528.8 & & \\
\hline
\end{tabular}

Tabla 5 Análisis ANOVA para el medidor 579AJ8 Fuente: Elaboración Propia

Los siguientes datos muestran el análisis en Minitab.

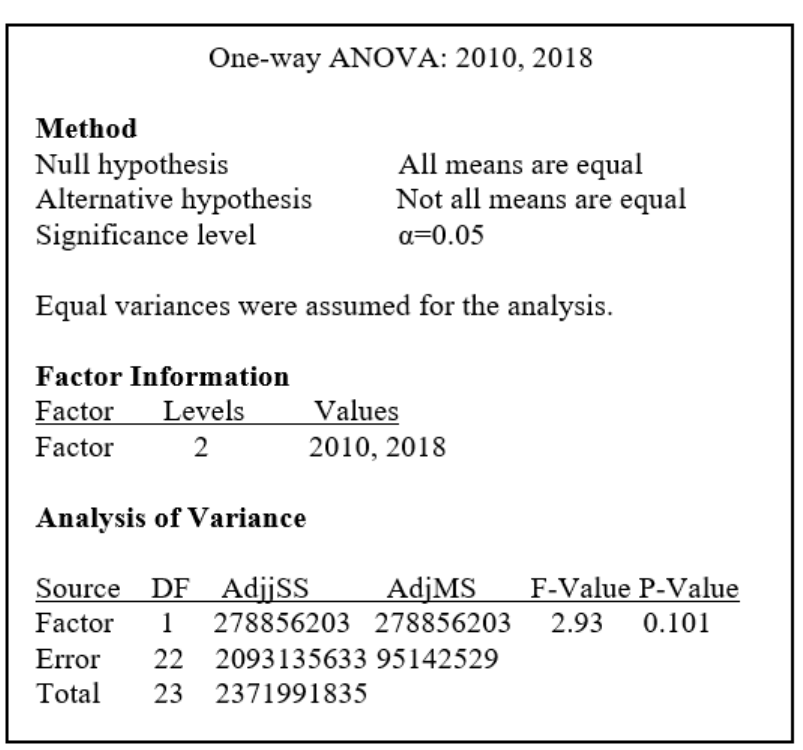

Cuadro 4 Análisis ANOVA para el medidor 579AJ8 en MINITAB

Fuente: Elaboración Propia

Se establecieron las hipótesis y al realizar el análisis de varianzas, se observó que la $\mathrm{F}$ calculada es menor que la $\mathrm{F}$ de tabla, además de que en el análisis en Minitab el p- valor es mayor a 0.05 , por lo tanto, no se rechaza la hipótesis nula, es decir no existe evidencia suficiente de que los monitores y ayudas visuales tengan un efecto en la disminución del consumo de energía eléctrica para ésta área del Tecnológico.

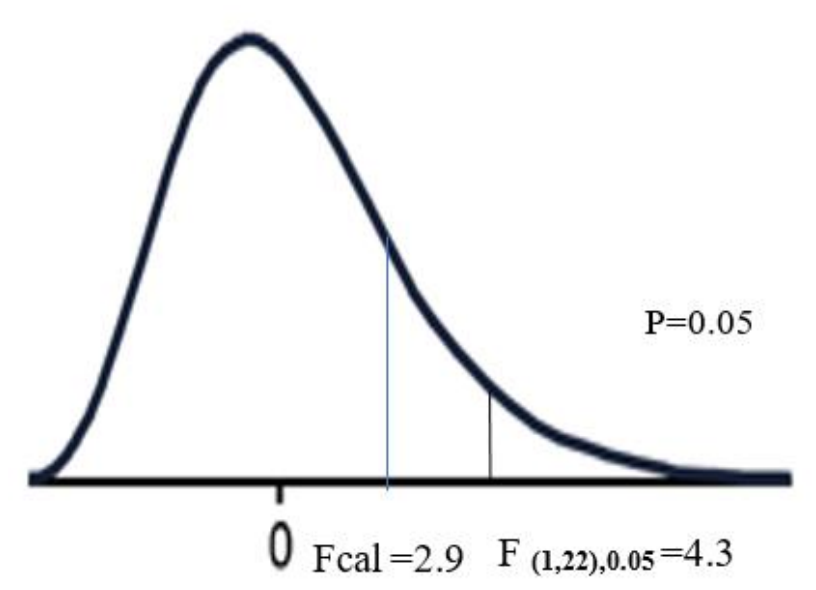

Figura 5 Distribución F para el medidor 579AJ8 Fuente: Elaboración Propia 


\section{Análisis de la distribución muestral de diferencia de medias}

\begin{tabular}{|c|c|c|}
\hline & Año 2010 & Año 2018 \\
\hline $\mathrm{n}$ & 12 & 12 \\
\hline $\bar{x}$ & 27322 & 20505 \\
\hline $\mathrm{s}$ & 11685.423 & 7330.479934 \\
\hline
\end{tabular}

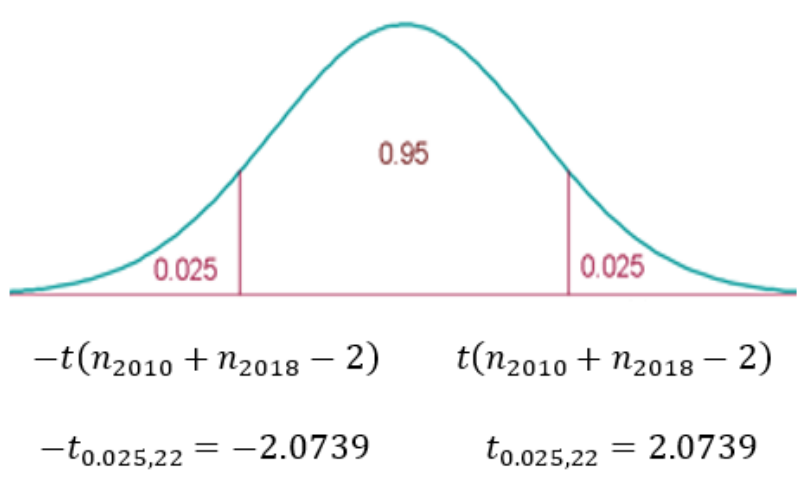

Figura 6 Distribución T-Student para diferencia de medias del medidor 579AJ8

Fuente: Elaboración propia

Ho; $\mu_{2010}=\mu_{2018}$

$H a ; \mu_{2010} \neq \mu_{2018}$

\section{Regla de decisión}

$$
\begin{aligned}
& H o ;-2.0739 \leq t c \leq 2.0739 \\
& H a ;-2.0739>t c \quad o \quad t c>2.0739 \\
& t_{c}=\frac{(27322-20505)}{\sqrt{(95142529)\left(\frac{1}{12}+\frac{1}{12}\right)}}=1.7119963 \\
& S C^{2}=\frac{\left(n_{2010}-1\right)\left(S_{2010}\right)^{2}+\left(n_{2018}-1\right)\left(S_{2018}\right)^{2}}{n_{2010}+n_{2018}-2} \\
& S C^{2}=95142529
\end{aligned}
$$

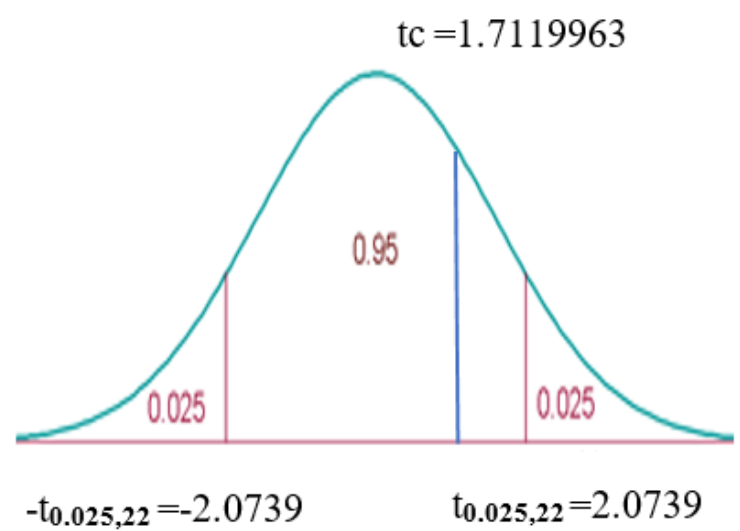

Figura 7 Comparación de valores de t de tabla, con la t calculada

Fuente: Elaboración Propia
$H_{o} ; \quad-2.0739 \leq 1.7119963 \leq 2.0739$

$H_{a} ; \quad-2.0739>1.7119963$

$1.7119963>2.0739$

Los datos muestran evidencia suficiente de que no existe efecto de los monitores y ayudas visuales en la disminución del consumo de energía eléctrica. Los siguientes datos muestran el análisis en Minitab de la prueba T.

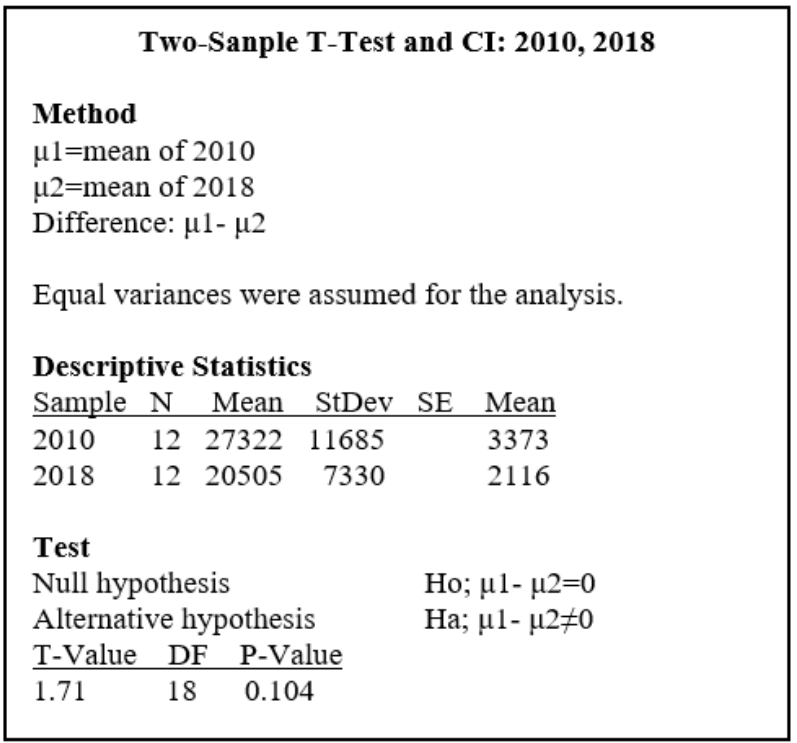

Cuadro 5 Análisis T-Student para el medidor 579AJ8 en MINITAB

Fuente: Elaboración Propia

\section{Análisis de Pruebas Pareadas}

$H o ; \mu_{D}=0$

$\alpha=0.05$

$\sum D=-81808$

$\frac{\alpha}{2}=0.025$

$\bar{D}=-6817$

$K=$ Diferencial esperada $=0$

$\sum D^{2}=1997233590$

$t_{\alpha / 2,(n-1)}=t_{0.025,11}=2.201$

$H o ; \mu_{D}$

$H a ; \mu_{D}$

$S_{D}^{2}=\frac{1}{n-1}\left(\sum D^{2}-\frac{\left(\sum D\right)^{2}}{n}\right)$

$S_{D}^{2}=130,865,562.2$

HERNÁNDEZ-RODRÍGUEZ, María Guadalupe, ORTEGA-CHÁVEZ, Laura Antonia, RUIZ-RODRÍGUEZ, Irma Judith y CARO-ESCUDERO, Iveth Selene. Control operacional para reducción de consumo de energía eléctrica en el Instituto Tecnológico de Chihuahua II. Revista de Aplicaciones de la Ingeniería. 2019. 
$S_{D}=11,439.64869$

$t_{c}=\frac{\sqrt{n} *(\bar{D}-k)}{S_{D}}$

$t_{c}=\frac{\sqrt{12} *(-17,567-0)}{26,049.742}=-2.06439$

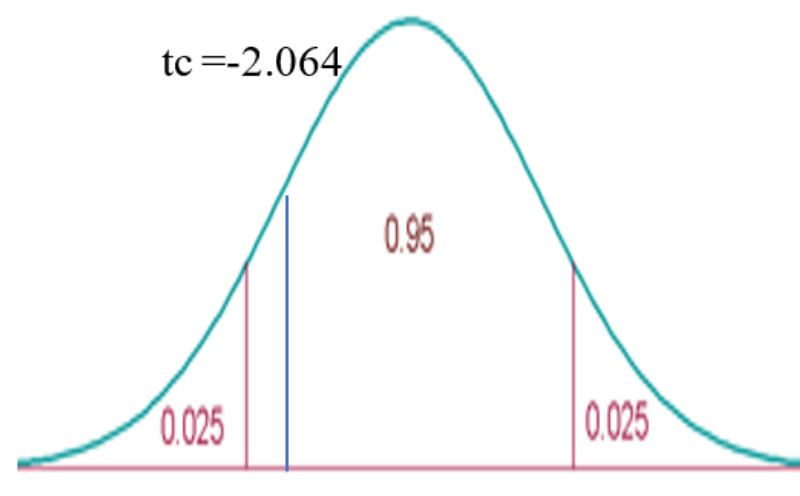

$\mathrm{t}=-2.201$

$\mathrm{t}=2.201$

Figura 8 Comparación de valores de t de tabla, con la $\mathrm{t}$ calculada para pruebas pareadas

Fuente: Elaboración Propia

Se acepta Ho, ya que $t_{c}>t_{\alpha / 2,(n-1)}$, es decir, existe evidencia suficiente para determinar que no hay efecto de los monitores y ayudas visuales para la disminución del consumo de energía eléctrica.

\section{Análisis de Pruebas Pareadas en Minitab}

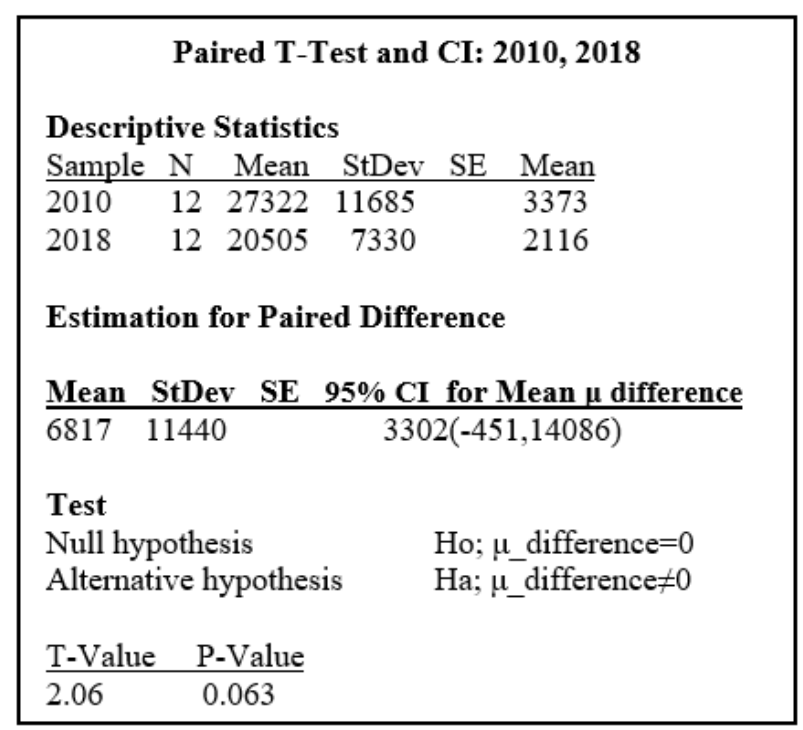

Con éste análisis se corrobora las decisiones tomadas con los anteriores métodos.

\section{Conclusiones}

Al observar los análisis realizados y los resultados que arrojaron, se puede concluir que la estrategia de los monitoreos y ayudas visuales para reducir el consumo de energía eléctrica, en el medidor Y574M8, está dando los resultados esperados, es decir hay una disminución significativa del consumo de energía eléctrica, a pesar de ser el área más extensa del Instituto Tecnológico de Chihuahua II, sin embargo, en el área controlada por el medidor 579AJ8 no está arrojando los resultados deseados, es decir no se está reduciendo el consumo de la energía eléctrica en forma significativa, lo cual implica buscar nuevas estrategias para esa área y reforzar las ya existentes para obtener mejores resultados, a su vez nos lleva a dar un paso más en la búsqueda de nuevas tecnologías que nos ayuden alcanzar nuestros objetivos, e iniciar nuevas investigaciones para los controles de consumos de energía eléctrica y reducción de las emisiones contaminantes al medio ambiente.

\section{Referencias}

Allen L. Webster, (2009). Estadística aplicada a los negocios y la economía

Astorga, Y. X. L. (2019). Diseño de una estrategia de comercialización para dispositivos solares: biosphare S.A. de C.V.

Infante y Zarate, (2012). Métodos Estadísticos: un enfoque multidisciplinario. Pag. 404-410

Manzini, F., J. Islas. M. Martinez. (2000). Reduction of Greenhouse Gases Using Renewable Energies in Mexico 2025. International Journal of Hydrogen Energy.

Navarro Chávez, J. C. L., Ortega, D., Odette, V., \& Díaz Pulido, A. (2019). La Eficiencia del Sector Eléctrico en México 2008-2015. Análisis económico, 34(85), 71-94.

Quevedo Héctor, Pérez Blanca. (2017). Estadística para Ingeniería y Ciencias, Grupo editorial Patria S.A. de C.V.

Richard C. Weimar, (2003). Estadística segunda edición.

Walpole. Myers. Myers, (2012). Probabilidad y estadística para ingeniería y ciencias, novena edición. 